\title{
An Employment of Probabilistic Neural Network in Magnetic Resonance Imaging For Early Brain Cancer Detection
}

\author{
Seba Aziz Sahy ${ }^{a}$ \\ ${ }^{a}$ Meddle Technical University, Institute Of Medical Technolgy Al-Mansour,Iraq \\ Saba.aziz@mtu.edu.iq
}

Article History: Received: 11 January 2021; Accepted: 27 February 2021; Published online: 5 April 2021

\begin{abstract}
Given the circumstances of the countries in which wars, political instability, and other uncertainties are passing that make the atmosphere impure, which have caused many diseases, one of these diseases that has spread widely is cancer. Cancer is a very common disease, and many of them affect a person and lead him or her to death. Among these diseases, which have been common in recent years specifically the brain tumors that they need early diagnosis and do not cause the death of the person. Furthermore, many studies in the field of brain cancer detection have been done, but the best solution is still missing. Therefore, in this paper, a reliable method is proposed to detect brain tumors, extract its properties, and classify the tumor using Magnetic Resonance Imaging (MRI) through the artificial neural network. In the proposed system, an essential part of image processing is the analysis and processing of digital images, especially to improve their quality, Bilateral Filter is used to improving image clarity and any image noise in this method preserves edges. After that, the distinctive properties of the image are extracted using the Histogram of Oriented Gradient (HOG) method. Thus, the extracted features are strong and can be classified as a Probabilistic Neural Network (PNN), this is what distinguishes our work from the previous works. The advantage obtained is granted to the PNN Classifier, which is used to train and test the accuracy of performance in perceiving the location of the tumour in MRI images of the brain accuracy as it resolves $99.5 \%$.
\end{abstract}

Keywords: Probabilistic Neural Network (PNN), Histogram of Oriented Gradient (HOG), Magnetic Resonance Imaging (MRI) Bilateral Filter.

\section{Introduction}

Diagnosing a brain tumor is important in the convincing treatment of this disease. The decision on the treatment method depends on the stage of the tumor at the time of the meeting, the type of pathology, and the size of the tumor. Computerized analytical methods like computer-aided diagnosis (CAD) confirms the nervous system by the experts. During neutrophils, the use of CAD combines the extensively studied [1]. Another research topic in this regard is the description of glioma, which is the main type of tumor [2].

The above-listed CAD systems are based identification, classification, and size of CAD-based brain tumors as a threat, and tumors have been on brain images with magnetic resonance imaging (MRI). This is due to the capacity of MRI to have a greater contrast to computed tomography (CT) images for soft tissues in the brain. The most recent developments in medical imaging focused on real-time detection of tumors using more accurate algorithms and the most active areas of research that have been discovered are brain MR tumors and CT scans. In biomedicine, MRI has been used to find the fine details internal structure of the body. This method is primarily used to discover tissue variations that have a good technique than CT. This allows MRI equipment a very unique tool for the identification of cancers and brain diagnosis [3]. Ionization computational chemistry is included and MRI utilizes a magnetic field to coordinate the nuclear magnetic polarity, and then the radio frequencies change the magnetization orientation that the scanner detects. This information can be processed further to generate additional data from the skin. The MR image is protected from automated imaging because it does not impact the body of a human. The main issues facing most medical imaging structures are the separation of cells and their nuclei from the rest of the photograph content. The process of separation, i.e. scalability is most important in creating an efficient clinical method.

Deep learning has a big role in Brain Cancer Detection and diagnosis [6]. Furthermore, among the deep learning techniques, the Probabilistic Neural Network (PNN) is the most effective classification techniques in brain cancer detection and diagnosis systems [7]. Subsequently, the employment of PNN in brain cancer detection and diagnosis as a complementary The discovery and extraction of tumors depend on classification algorithms, whether supervised or unsupervised; although each class of classification for both groups has its basic characteristics and principles [4]. Good treatment is given through the classification process that is giving the correct decision. The image of the entrants selected must be divided into two classifications, i.e. the ordinary and the unnatural. Categorization is performed using tumor characteristics that contain both images and normal images [5]. part of the proposed systems could give better results [8].

In this work, classification of brain MRI has been performed utilizing PNN and discovery of tumor utilizing image information that enters the fragmentation of the image into groups of features. More analysis can be done quickly if the same features are derived from the MRI. The extraction of features plays an important part in deciding a workbook's output. The division is performed on the leafy image after classification to extract the tumor region.

This work is segmented into seven sections, starting with the Introduction. Section 2 discusses the most related work for early brain cancer detection. Section 3 explains the employment of Probabilistic Neural Network (PNN) in brain cancer detection. Section 4 demonstrates the evaluation methods which have been used to evaluate the performance of the proposed method. Whereas, the design and architecture of the proposed method have been 
explained in Section 5. Furthermore, section 6 provides the obtained results, and Section 7 presents the conclusion of the work.

\section{Related Work}

There are many well-established studied in the field of brain cancer detection. In this section, the range of research that has been used to classify brain cancer tumors will be reviewed as shown below:

In [5] authors have proposed a comparative method for identifying brain tumors. Their approach included image mining techniques like (GLCM) Gray Level Cooccurrence, Intensity-based Histogram features, Intensity-based features. The techniques were applied to the BraTS database which showed good results in terms of accuracy. The techniques GLCM, Intensity- features were based Histogram features, Intensity-based compared with the J48 algorithm. GLCM showed 95.25 in accuracy whose value is very close to the J48 algorithm.

Previous work [6] demonstrates a non-controlled approach of reading from the MRI images to segment brain tumors. This technique has shown better results than the techniques of the registry and DNN. In addition, to overcome the problem of call errors in the audio-based technique (labeling) to infer a probability system using the EM algorithm, an actual persistent (undetermined) image poster can be obtained if the probability that the latent images we identify are controlled by the distribution. The DNN model turned into blended with the EM set of rules to teach this probabilistic version. While zoomed in, the wrong label is restored to the underlying image. This approach was tested in two extraordinary datasets, the first being well-known open MR photographs registered within the Internet mind segmentation facts warehouse (IBSR) and marmoset mind statistics dataset.

To increase CNN accuracy for the segmentation of mind tumors [7], Hybrid wavelet transformation SWT and GCNN were suggested. SWT was utilized to extract features that give a better result to discontinuous data from the Fourier transform method. After extracting the features, Random Forest Classifier was used for fragmentation and then they utilized GCNN to train the model. The proposed method yielded $2 \%$ improvement in PSNR and SSN over conventional CNN [8].

Toğaçar's previous work [9] proposed a new CNN model called Brain MRNet. The basic design of the model is based on attention units and a hyper-segment strategy. With this method, the highlights removed from each level of the Brain MRNet model are kept in the exposure structure at the last level. The point is to choose the best and most qualified highlights from the highlights that the exhibition maintains. The Brain MRNet model is more fruitful than the prefabricated convolutional nervous system models used in this study (AlexNet, GoogleNet, VGG-16). The result of the grouping carried out with the Brain MRNet model was $96.05 \%$.

Maharjan et al. [10] proposed a technique designed to extend the precision of order by reducing the risk of an additional problem and supporting multitask classification. The proposed framework consists of restraint of the nervous system with maximum regulated capacity and control. The accuracy and preparation time of the different types of tumors were determined. Probability point of labeled data and time of execution. A special accuracy plan and processing time were obtained by testing the proposed framework using various samples of MRI images. The result shows that the proposed arrangement is superior to different frameworks. In addition, the proposed arrangement has improved accuracy of almost $2 \%$ and a smaller installation time of 40-50 milliseconds compared to other existing arrangements and solutions.

However, according to the above discussion, it is observed that the employment of PNN as a complement part in classification could give better performance and increase the accuracy of brain cancer detection and diagnosis. Therefore, in this work, the PNN has been employed for classification brain cancer from the images.

\section{The Probabilistic Neural Network (PNN)}

The PNN is a neural model with an oblique diagonal basic network, adapted to classification problems and based on Bayes' decision-making principle, and also based on the Pazen window and its probability density function (PDF) for each class is based on training examples [9] [10]. The performance of this neural model has a rapid learning speed in the recognition properties of small contraction components and can easily add new typical neural cells to the pattern layer. Unfortunately, PNN requires a large amount of memory because the model nodes in the patterns are the same as all training samples, which generally depend on the sample size and cause a large network size [11].

\subsection{Architecture of Probabilistic Neural Network (PNN)}

The model structure consists of a multilayer feed network. It uses a supervised learning training method and included four layers: the input layer, the pattern layer, the summation layer, and the output layer [12]. However, the architecture of the PNN is consists of three-layer which are the input layer, hidden layer, and class layer as shown in Fig. 1. 


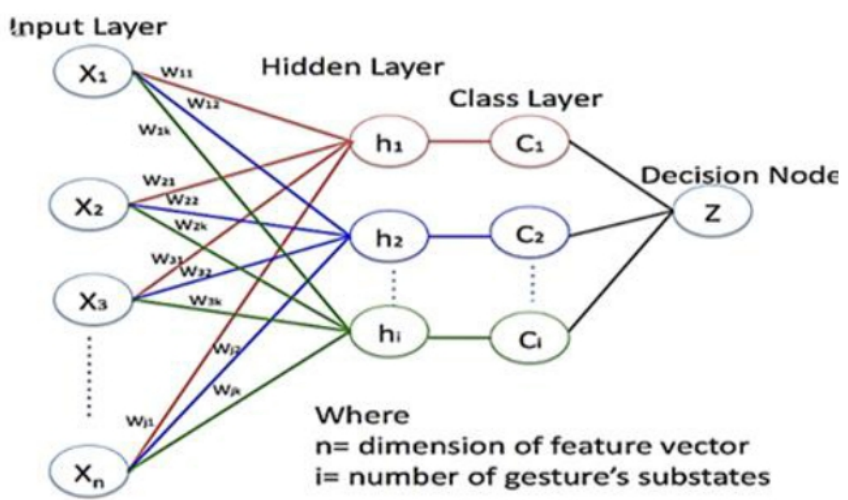

Fig. 1. Probabilistic Neural Network Architecture [12].

In the input layer that is completely connected to the etching layer, the input feature vector $(\mathrm{x})$ is distributed across all nerve nodes in the pattern layer. The pattern layer contains one neuron per training sample. In the model layer, each neuron node calculates the distances between the input feature vector and training samples and produces a vector whose elements indicate how close the inputs are to the training sample and any distance scale such as Euclidean Distance, Euclidean Square Distance, and Manhattan Distance that can be used to calculate the distance [13], [14]. All neurons perform a Gaussian function (radial transfer function) that can be simplified as follows:

$$
\mathrm{G}(\mathrm{x})=\exp \left(-\mathrm{D} /\left(2 \sigma^{\wedge} 2\right)\right)
$$

Wherein, $\mathrm{G}(\mathrm{x})$ represents the output of the neuron pattern node, and $\mathrm{X}$ is the input feature vector of the $\mathrm{Ci}$ category. Whereas D represents the distance between the input features vector and the chart vector belonging to a specific class (Euclidean distance as shown in Eq. (2) was used in this work), and $\sigma$ is the smoothing factor.

Neurons in the same layer belonging to the same nerve grouped neuron. In the plural layer, there is one nerve node for each class, and the pattern layer outputs for each class are combined and produce outputs representing the probability of the classes (obtaining and estimating the probability density function for each class), as shown in Eq. (3).

Wherein, $\mathrm{O}(\mathrm{x})$ represents the output of summation node I for class $\mathrm{Ci}, \mathrm{nj}$ is the number of samples in the pattern layer of class $\mathrm{Ci}$, and $\mathrm{G}(\mathrm{x})$ is the output of the pattern $\mathrm{j}$ node.

The output layer (decision layer) picks the maximum one of these probabilities, as given in Eq. (4):

Target $\operatorname{class}(\mathrm{x})=\operatorname{maximum}(\mathrm{O}(\mathrm{X}))$

It remains the decision that the smoothing factor is assigned to the Gaussians. The value of $\sigma$ affects the results of recognition of PNN workbooks, and they must be chosen with care. More than one algorithm can be used to define the scope of the smoothing element. In this work, the minimum and maximum values of the standard deviation after the average vector of each class are used to determine the range. In any field of science, it is almost a requirement that the performance of a classifier must be evaluated to understand and measure the suitability of classifying a given problem. The classification of binary predicts all instances of data for the test data set either negative or positive. This classification or prediction results in four outcomes - true or false (positive and negative). Furthermore, several evaluation methods have been used to evaluate the performance of the proposed methods. In this work, one of the most common measures which is the confusion matrix is used.

\section{The proposed Method}

In this section, the architecture of the proposed system has been presented. Furthermore, the proposed method consists of subsequent stages: first stage acquisition of the image, second stage extraction of brain cancer features, selection of more suitable features, classification to determine the correct category of the brain used precise identification of PNNs depends primarily on careful selection of features. This PNN is utilized as a classifier to distinguish the brain shown in this Fig. 2. 


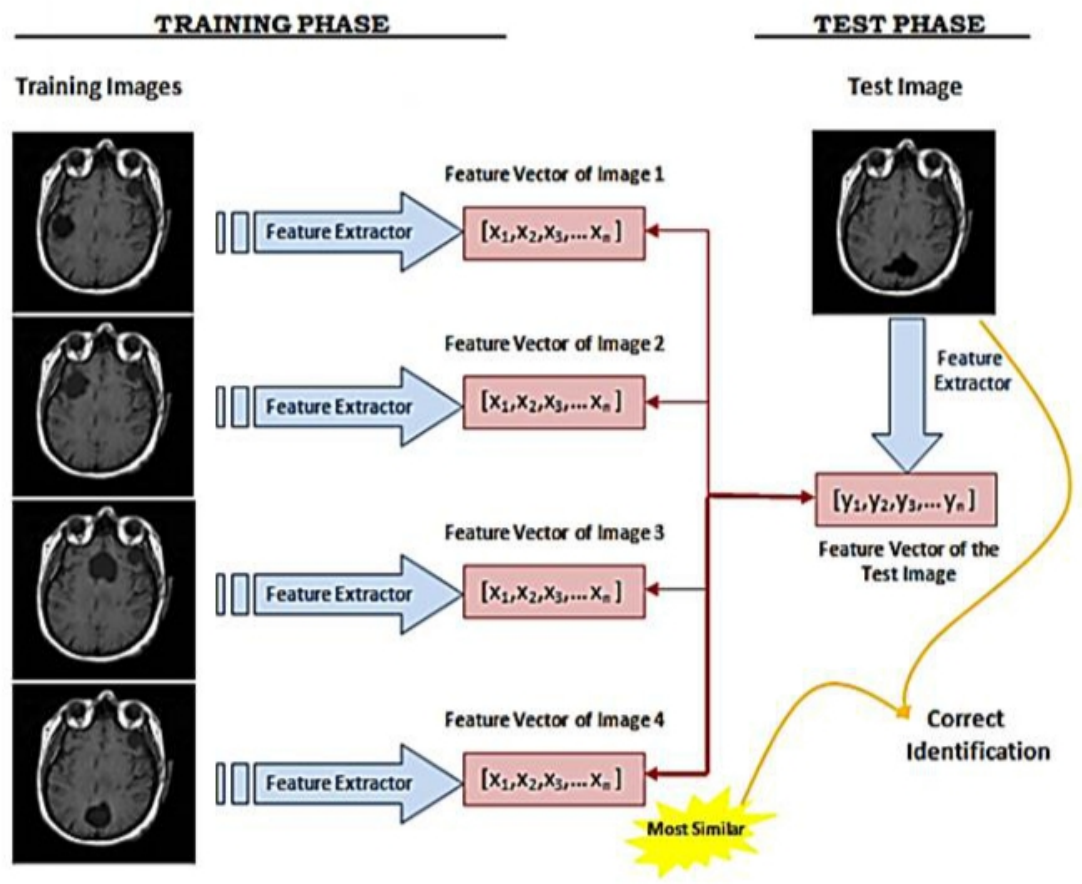

Fig. 2. The Main Proposed System.

\subsection{Pre-processing stage using Bilateral Filter}

This improved Gaussian method was used, because of problems when Gaussian used a unit, the filters were improved by doubling the use of another Gaussian filter due to multiple iterations and different pixel density i.e. [19], meaning that pixels with a density similar to the ones in the middle are included only to calculate an intense density value.

As a result, this technique preserves the edges, because the adjacent pixels placed on the other side of the edge for pixels are closer to the edges and therefore large differences in density appear blur when compared to the central pixel.

\subsection{Histogram of Oriented Gradient (HOG) feature descriptor}

In this system, the Histogram Oriented Gradient (HOG) algorithm is used for brain cancer, and the additional benefit of using this algorithm is that it will help extract image features. This will assist in training and testing the PNN [20], [21]. HOG extracts the color feature, texture feature, and shapes the context feature counts occurrences of gradient orientation in localized portions of an image - detection window. These features are stored in a mat file and used for PNN training.

\subsection{PNN Classification}

In this paper, we have trained PNN from scratch to apply. The network layers of PNN work as a candidate for presence detection from specified features or patterns that exist in the image. The first layers of PNN have detected a lot of features that can explain easily. In the second layer, the layers uncover littler features that are progressively unique. The last layer is the ability to produce too detailed classification by incorporating all features discovered in previous layers. PNN inputs are MR brain images. Each neuron calculates the product of a point of weights to the local area it is connected to the volume. However, the dataset is segmented into two stages which are training stage and testing stage. Furthermore, $70 \%$ of the images have been used to train the proposed system, whereas, $30 \%$ of the images have been used to test the proposed system.

Experimental Results

This method for detecting brain cancer works best by deep learning, which gives very accurate results because it works at more than one level and after that, a decision is made after entering the strong fevers that were extracted through HOG and this is explained through the results shown below.

\subsection{Pre-processing stage:}

This is the important stage during which the original image passes through the process of initialization through the use of the enhanced filter, through which we obtain a clear image empty of noise and with prominent edges, as shown in Fig. 3. 


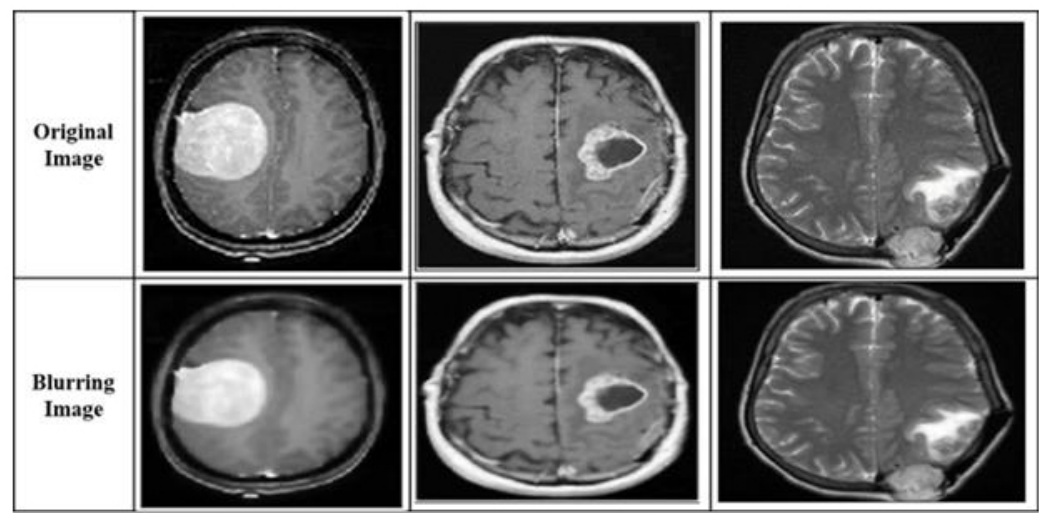

Fig. 3. Blurring image.

\subsection{Feature Extraction using HOG Algorithm}

After pre-processing, feature descriptors are described in points of interest in both images, and this is the step by which the descriptor is calculated based on the areas centred on the detected features. This includes converting the local pixel neighbourhood into a small vector representation that allows comparison of neighbourhoods regardless of changes in brain orientation or scale as shown in Figure 4.

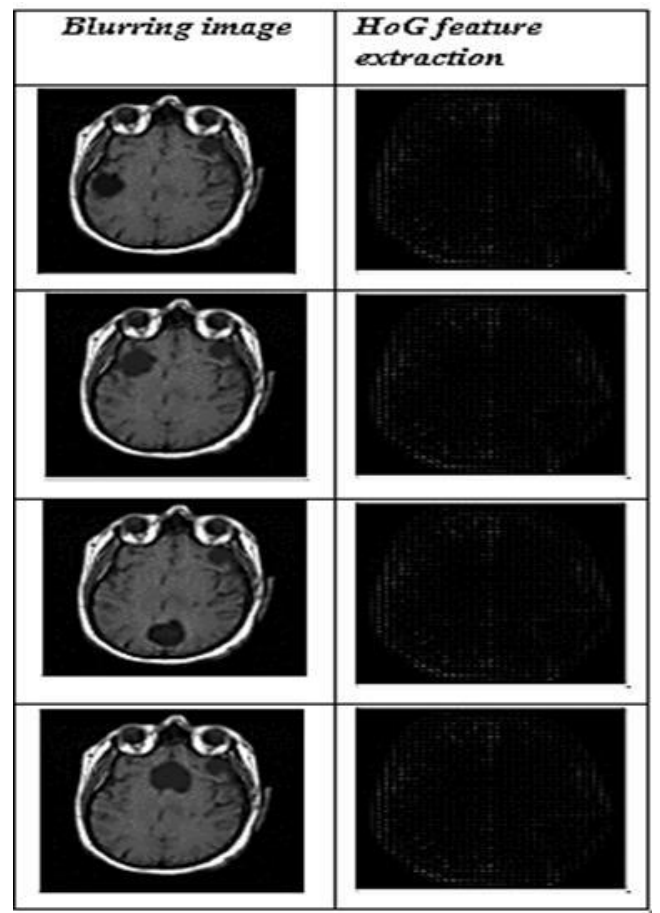

Fig. 4. Feature Extraction using the HOG algorithm.

Table (1) shows a sample of extraction of features by the HOG method, where each column represents its value to extract five vectors $(\mathrm{x}, \mathrm{y}, \mathrm{o}, \mathrm{s}, \mathrm{d})$ represents location, scale, orientation, and Descriptor.

Table (1): Feature Vector of HOG.

\begin{tabular}{|l|l|l|l|l|}
\hline $\mathrm{X}$ & $\mathrm{Y}$ & Orientation & Scale & Descriptor \\
\hline 8045.728 & 9.07515 & 0.010613 & -0.00131 & 7.95458 \\
\hline 11390.78 & 7.565268 & 0.00742 & 0.00307 & 7.922516 \\
\hline
\end{tabular}




\begin{tabular}{|l|l|l|l|l|}
\hline 6571.419 & 9.954212 & 0.019222 & 0.002761 & 7.84858 \\
\hline 7574.995 & 9.33698 & 0.017185 & 0.001503 & 7.773198 \\
\hline 9622.515 & 8.297918 & 0.008062 & 0.002341 & 7.814751 \\
\hline 6229.719 & 10.18612 & 0.011815 & 0.00509 & 7.883937 \\
\hline 9644.994 & 8.287784 & 0.010464 & 0.004348 & 7.859967 \\
\hline 6575.027 & 9.951828 & 0.016399 & 0.000801 & 7.755506 \\
\hline 7895.692 & 9.156901 & 0.014915 & 0.002644 & 7.910714 \\
\hline 7543.508 & 9.35507 & 0.012684 & 0.00338 & 7.914761 \\
\hline 7665.403 & 9.285454 & 0.011812 & 0.004628 & 7.955516 \\
\hline 9435.419 & 8.383192 & 0.009911 & 0.002045 & 7.957663 \\
\hline 9178.776 & 8.502956 & 0.011363 & 0.005182 & 7.929629 \\
\hline 10437.47 & 7.944853 & 0.009457 & 0.001667 & 7.844263 \\
\hline
\end{tabular}

\subsection{PNN Classification:}

Based on the obtained results, it is observed that the proposed method achieved an excellent result with an accuracy of $99 \%$, sensitivity of $96 \%$, and specificity of $99 \%$. Furthermore, the result of PNN detection of brain cancer is divided into two phases normal and abnormal. Table 3 shows the obtained results of the proposed method.

\section{Evaluation Methods}

The Confusion matrix consists of $2 \times 2$ Tables that contain four results that are produced as a classification, these are essential performance measures, like accuracy, specificity, and sensitivity which are derived from the confusion matrix. The confusion matrix is utilized to represent the test of the result prediction model [14], [15], and [16]. Each of the rows stands for the predicted class which means the (Output Class), and the columns stand for the true class which means the (Target Class). In Table (2), the matrix of confusion is displayed, which is described as the different values and equations associated with them. Few of these equations are closely related to performance analysis.

Table (2): A Typical Confusion matrix [14].

\begin{tabular}{|l|l|l|l|}
\hline \multicolumn{2}{|l|}{ Confusion Metrix } & \multicolumn{2}{l|}{ Predicted } \\
\cline { 3 - 4 } & Negative & Positive \\
\hline \multirow{2}{*}{ Actual } & Negative & TN & FP \\
\cline { 2 - 4 } & Positive & FN & TP \\
\hline
\end{tabular}

The inputs of the confusion matrix have meaning in the context of the problem of data mining [17], [18]:

$\mathrm{TN}$ is the number of the prediction of true that is the negative case, 
$\mathrm{FN}$ is the number of predictions of false that a case positive,

TP It is a valid forecast number with a positive example,

$\mathrm{FP}$ is the number of negative false predictions.

Furthermore, the following are the Basic measures derived from the confusion matrix:

Accuracy

ACC is determined as a number for all predictions of correct $(\mathrm{TP}+\mathrm{TN})$ divided by the total number of data sets $(\mathrm{P}+\mathrm{N})$. The best accuracy equal to 1.0 , while the worst equal to 0.0 . It can likewise be determined by 1 error (ERR) as shown in Eq. (1) [16].

(5)

$$
\mathrm{ACC}=(\mathrm{TP}+\mathrm{TN}) /(\mathrm{TP}+\mathrm{TN}+\mathrm{FN}+\mathrm{FP})=(\mathrm{TP}+\mathrm{TN}) /(\mathrm{P}+\mathrm{N})
$$

\section{b. Sensitivity}

Calculated the number of true positive (TP) predictions divided by a total of positive (P) predictions of this method called Sensitivity (SN) or likewise Recall or the True Positive Rate (TPR) (REC). The sensitivity equal to 1.0 is best, whereas the worst equal 0.0 as shown in Eq. (2) [16].

$\mathrm{SN}=\mathrm{TP} /(\mathrm{TP}+\mathrm{FN})=\mathrm{TP} / \mathrm{P}$

c. Specificity (True Negative Rate)

Calculated the number of True Negative (TN) predictions divided by the total the number of negatives $(\mathrm{N})$ this method is called Specificity or True Negative Rate (TNR). The specificity equal to 1.0 is best, whereas the worst equal 0.0 as shown in Eq. (3) [16].

$$
\mathrm{Sp}=\mathrm{TN} /(\mathrm{TN}+\mathrm{FP})=\mathrm{TN} / \mathrm{N}
$$

However, the proposed model is limited to the sensitivity in allocating all cancer areas. Table (3) shows the number of images, classes, sample, and the performance of the proposed model based on the criteria of Sensitivity, Specificity, and Accuracy.

Table (3) Evaluation performance of PNN.

\begin{tabular}{|l|l|l|l|l|l|}
\hline N0. & Class & samples & Sensitivity & Specificity & Accuracy \\
\hline Image 1 & Class1 & 150 & 0.97 & 0.98 & 0.98 \\
\hline Image 2 & Class2 & 150 & 0.95 & 0.98 & 0.99 \\
\hline Image 3 & Class3 & 150 & 0.96 & 0.99 & 0.99 \\
\hline Image 4 & Class4 & 150 & 0.99 & 0.99 & 0.99 \\
\hline Image 5 & Class5 & 150 & 0.93 & 0.99 & 0.99 \\
\hline Overall & & 0.96 & 0.99 & 0.99 \\
\hline
\end{tabular}

Finally, according to the obtained results, it is observed that the proposed model is effective in brain cancer detection. Subsequently, the obtained results have been compared with the related work of Kumar [5] and Lundervold [6] and our proposed model achieved the highest performance with an accuracy of $99 \%$ as shown in the bellow table. 
Table (3) The comparison of proposed model with the related work.

\begin{tabular}{|l|l|l|}
\hline \multicolumn{1}{|c|}{ References } & Algorithm & Accuracy \\
\hline Kumar at.al [5] & Gray Level Co-Occurance & 95.25 \\
\hline Lundervold [6] & DNN & $98 \%$ \\
\hline Our Proposed Model & PNN & $99 \%$ \\
\hline
\end{tabular}

\section{Conclusions}

In this paper, we presented a new method for PNN to classify the most common types of brain tumors. This technique requires pre-processing steps using an improved method Bilateral Filter and then the important points are extracted by image using HOG and the extracted feature are used classified by PNN, this group of images has a better classification of tumor pixels that can be obtained and will increase the degree of accuracy as it resolves $99 \%$. Our suggested method may be implemented as a simple and useful tool for doctors in segmenting brain tumors in MRI images. The propsed model is limited to the sensitivity in allocating all cancer areas. In the future work, deffrient classifier is required for classification stage, also, test the proposed system with a large number of images is recommended.

\section{Abbreviations}

MRI Magnetic Resonance Imaging

HOG Histogram of Oriented Gradient

PNG Probabilistic Neural Network

CAD Computer-Aided Diagnosis

1. Arunkumar, N.; Mohammed, M.A.; Ghani, M.K.; Ibrahim, D. A.; Abdulhay, E.; Ramirez-Gonzalez, G.; and de Albuquerque, V.H. (2019). K-means clustering and neural network for object detecting and identifying abnormality of brain tumor. Soft Computing, 23(19), 9083-9096.

2. Shakeel, P.M.; Tobely, T.; Al-Feel, H.; Manogaran, G.; and Baskar, S. (2019). Neural network based brain tumor detection using wireless infrared imaging sensor. IEEE Access, 7, 5577-5588.

3. Abir, T.A.; Siraji, J.A.; Ahmed, E.; and Khulna, B. (2018). Analysis of a novel MRI based brain tumour classification using probabilistic neural network (PNN). Int. J. Sci. Res. Sci. Eng. Technol., 4(8), 65-79.

4. Kavitha, A.R.; and Chellamuthu, C. (2017). Brain tumour segmentation and detection using modified region growing and genetic algorithm in MRI images. International Journal of Medical Engineering and Informatics, 9(3), 269-283.

5. Kumar B.S. and Selvi R.A., "Feature extraction using image mining techniques to identify brain tumors, " in 2015 International Conference on Innovations in Information, Embedded and Communication Systems (ICIIECS), 2015, pp. 1-6.

6. Lundervold, A.S. (2019). An overview of deep learning in medical imaging focusing on MRI. Zeitschrift für Medizinische Physik, 29(2), 102-127.

7. Nayak, D.R.; Dash, R.; Majhi, B.; and Prasad, V. (2017). Automated pathological brain detection system: A fast discrete curvelet transform and probabilistic neural network based approach. Expert Systems with Applications, 88, 152-164.

8. Dahab, D. A.; Ghoniemy, S. S.; and Selim, G. M. (2012). Automated brain tumor detection and identification using image processing and probabilistic neural network techniques. International journal of image processing and visual communication, 1(2), 1-8.

9. Liu, F.; Zhou, Z.; Samsonov, A.; Blankenbaker, D.; Larison, W.; Kanarek, A.; and Kijowski, R. (2018). Deep learning approach for evaluating knee MR images: achieving high diagnostic performance for cartilage lesion detection. Radiology, 289(1), 160-169.

10. Toğaçar, M.; Ergen, B.; and Cömert, Z. (2020). Brain MRNet: Brain tumour detection using magnetic resonance images with a novel convolutional neural network model. Medical Hypotheses, 134, 109531 
11. Maharjan, S.; Alsadoon, A.; Prasad, P.W.; Al-Dalain, T.; and Alsadoon, O. H. (2020). A novel enhanced softmax loss function for brain tumour detection using deep learning. Journal of neuroscience methods, 330, 108520.

12. Mason, C.; Twomey, J.; Wright, D.; and Whitman, L. (2018). Predicting engineering student attrition risk using a probabilistic neural network and comparing results with a backpropagation neural network and logistic regression. Research in Higher Education, 59(3), 382-400.

13. Sulaiman, S.N.; Non, N.A.; Isa, I.S.; and Hamzah, N. (2014). Segmentation of brain MRI image based on clustering algorithm. In proceeding of the first Symposium on Industrial Electronics \& Applications (ISIEA). Kota Kinabalu, Malaysia, 60-65.

14. Singh, A. (2015). Detection of brain tumor in MRI images, using combination of fuzzy c-means and SVM. Proceeding of the second International Conference on Signal Processing and Integrated Networks (SPIN). Noida, India, 98-102.

15. Hu, K.; Gan, Q.; Zhang, Y.; Deng, S.; Xiao, F.; Huang, W.; and Gao, X. (2019). Brain Tumor Segmentation Using Multi-Cascaded Convolutional Neural Networks and Conditional Random Field. IEEE Access, 7, 92615-92629.

16. Mohan, G.; and Subashini, M.M. (2018). MRI based medical image analysis: Survey on brain tumor grade classification. Biomedical Signal Processing and Control, 39, 139-161.

17. Amin, J.; Sharif, M.; Yasmin, M.; and Fernandes, S.L. (2017). A distinctive approach in brain tumor detection and classification using MRI. Pattern Recognition Letters.

18. Deepak, S.; and Ameer, P.M. (2019). Brain tumor classification using deep CNN features via transfer learning. Computers in biology and medicine, 111, 103345.

19. Mustafa, W.A.; and Yazid, H. (2016). Illumination and contrast correction strategy using bilateral filtering and binarization comparison. Journal of Telecommunication, Electronic and Computer Engineering (JTEC), 8(1), 67-73.

20. Mittal, M.; Goyal, L.M.; Kaur, S.; Kaur, I.; Verma, A.; and Hemanth, D. J. (2019). Deep learning based enhanced tumor segmentation approach for MR brain images. Applied Soft Computing, 78, 346-354.

21. Nimbhorkar, N.B.; and Alaspurkar, S.J. (2014). Probabilistic neural network in solving various pattern classification problems. IJCSNS International Journal of Computer Science and Network Security, 14, 133-137. 